\title{
Intraoperative Ultrasonography in Resection of Diffuse Glioma
}

\author{
Sayedali Ahmadii* ${ }^{*}$, Said Safari ${ }^{\circledR}{ }^{\circledR}$, Mojtaba Chardoli $^{3}$, Marjan Mirsalehi ${ }^{4}$ \\ 'Department of Neurosurgery, Iran university of Medical Sciences, Tehran, Iran \\ ${ }^{2}$ Functional Neurosurgery Research Center, Shohadaye Tajrish Comprehensive Neurosurgical Center of Excellence, Shahid \\ Beheshti University of Medical Sciences, Tehran, Iran \\ ${ }^{3}$ Iran University of Medical Sciences, Firoozgar General Hospital Emergency Department, Tehran, Iran \\ ${ }^{4}$ ENT and Head \& Neck Research Center and Department, the Five Senses Health Institute, Iran University of Medical \\ Sciences, Tehran, Iran
}

*Correspondence to Sayed Ali Ahmadi, M.D., Department of Neurosurgery, Iran University of Medical Sciences,

Tehran, Iran. Tel: +98(21)66503890; Fax: +98(21)66503890; Email: ahmadi.sayedali@gmail.com, ahmadi.as@iums.ac.ir

Published online March 27, 2021

\section{Dear Editor,}

Diffuse glioma is a primary brain tumor that originates from glial supportive cells and is the most common type of intra-axial brain tumor. Open tumor resection is the first step in the treatment of diffuse gliomas. The extent of resection (EOR) directly influences clinical outcomes in glioma surgery. However, resection of diffuse glioma is encountered with the problem of macroscopic and even microscopic similarity of normal brain and tumor. Furthermore, maximizing EOR requires attention because a mistake in identifying and preserving normal brain regions in complete resection can lead to catastrophic complications.

Some tools such as intraoperative magnetic resonance imaging (MRI), intraoperative ultrasonography (IOUS), and fluorescence-guided microsurgery are used in glioma surgery with the goal of maximizing the EOR. IOUS is more available and is inexpensive compared to other intraoperative imaging modalities. ${ }^{1}$

In glioma surgery the first IOUS scan can be performed after craniotomy before the dura mater is opened; at this time brain-shift is not yet created and images can compare to preoperative images. IOUS can localize the glioma and define its margins, dimensions, morphology, and echogenic features. Additionally, anatomical landmarks such as falx, tentorium, ventricles, skull base, and major vessels can be identified. IOUS provides real-time imaging of the glioma in an attempt to bypass the shift of tissues that occurs throughout the surgery. High frequency twodimensional IOUS (2D-IOUS) can distinguish between peritumoral parenchymal edema and the tumor boundary in diffuse glioma.

IOUS can be used just as a navigator to determine the best route to make access to the lesion and avoid the injury to the normal brain, also IOUS can be applied for real-time IOUS can be used to control sufficient tumor resection.

Navigated intraoperative ultrasound (NIOUS) is a powerful tool that combines the benefits of real-time $2 \mathrm{D}$ ultrasound (2D-US) imaging with the ability to track the lesion by US probe, allowing for image-guided Navigation. NIOUS can be used alone (direct NIOUS [DNIOUS]) or it can be used in combination with preoperative MRI (image fusion mode). By the combination of the frameless navigation with $3 \mathrm{D}$-ultrasound, the most prominent drawback of $2 \mathrm{D}$-ultrasound and conventional neuronavigation (the orientation and brain shift) were solved. Fusion imaging of -re-operative MRI and IOUS is very useful to detect poor sonographic visibility tumors on the B-mode US. Fusion images may demonstrate multiplane images including the same images of the MRI and US to help localize the tumor. ${ }^{2}$

Contrast-enhanced ultrasonography (CEUS) is another modality providing a real-time dynamic and continuous view of vascularization and flow distribution patterns to differ between a normal brain and diffuse glioma. CEUS is a fast, safe, dynamic, and real-time tool that is helpful during surgery in differentiating malignant and benign gliomas from the normal brain and thus deciding a surgical strategy. ${ }^{3}$

IOUS was helpful in identifying nearby vascular structures using Doppler angiography sonography. These vessels can be visualized by switching into color Doppler sonography mode. In 3D-IOUS powered Doppler identifies the arteries by fusion of the real-time US imaging by pre-operative MR-angiography. A good correlation between IOUS images and those of intraoperative MRI has also been shown in diffuse glioma surgery. 
There are many studies that clarify the role of real-time IOUS in total resection of diffuse gliomas, and its ability to identify non-enhancing residual tumor tissue, may improve prognosis and survival. In conclusion, IOUS is easily available, inexpensive, convenient, fast, and easy to use and may provide a more practical alternative setting in diffuse glioma surgery.

\section{Conflict of Interest}

The authors declare that they have no conflict of interests.

\section{Ethical Statement}

Not applicable.

\section{References}

1. Garzon-Muvdi T, Kut C, Li X, Chaichana KL. Intraoperative imaging techniques for glioma surgery. Future Oncol. 2017;13(19):1731-45. doi: 10.2217/fon-2017-0092.

2. Jenkinson MD, Barone DG, Bryant A, Vale L, Bulbeck H, Lawrie TA, et al. Intraoperative imaging technology to maximise extent of resection for glioma. Cochrane Database Syst Rev. 2018;1(1):CD012788. doi: 10.1002/14651858. CD012788.pub2.

3. Wang J, Yang Y, Liu X, Duan Y. Intraoperative contrast-enhanced ultrasound for cerebral glioma resection and the relationship between microvascular perfusion and microvessel density. Clin Neurol Neurosurg. 2019;186:105512. doi: 10.1016/j. clineuro.2019.105512.

Citation: Ahmadi S, Safari S, Chardoli M, Mirsalehi M. Intraoperative ultrasonography in resection of diffuse glioma. Clin Neurosci J. 2021;8(2):51-52. doi:10.34172/ icnj.2021.11. 